\title{
Model Based Optimisation of a Step in Acceleration for a CR-Diesel Engine
}

\author{
Heiko Sequenz, Matthias Mrosek, Simon Zydek, Rolf Isermann \\ Institute of Automatic Control, Laboratory of Control Engineering and \\ Process Automation, Technical University Darmstadt, Germany \\ (e-mail: hsequenz@iat.tu-darmstadt.de)
}

\begin{abstract}
Due to more and more stringent emission regulations the optimisation of transient engine operation becomes necessary for future Diesel engine developments. Exemplary for the transient operation a step in acceleration pedal is investigated and optimised with respect to the engine emissions. This gives insights about the emission formation at transient driving and a benchmark for the design of open and closed-loop controls. A model structure to simulate the engine emissions and the engine torque is presented. It consists of a dynamical mean value air path model and a stationary combustion model. Thus the relevant outputs $\mathrm{NO}_{\mathrm{x}}$, soot and torque are simulated with respect to the engine actuators. For optimisation the calibrated emission values for steady state are regarded. The actuators of the air path and the crank angle of the main injection are optimised such that the emissions follow a desired trajectory. The results are compared to a non-optimised step in acceleration and to a nonlinear closed loop control of the air path.
\end{abstract}

Keywords: Emission Models, Air Path Models, Optimisation.

\section{INTRODUCTION}

During the engine development process a great effort is taken to optimise the Diesel engine's fuel consumption while meeting the legislative emission boundaries. The optimisation for steady state conditions can be regarded as state of the art. However the emitted emissions during transient driving differ highly from the calibrated steady state emissions. Figure 1 shows the emissions for a ramp in injection. The soot emissions are shown for a dynamic simulation and for a quasi-stationary simulation assuming that the dynamics are decayed infinitely fast. In order to fulfil future emission standards, the consideration of transient emissions becomes more and more necessary. Due to the multiobjective optimisation criterion already for steady state conditions a systematical optimisation on the testbed is highly time consuming. For the transient emissions further problems arise from significant sensor dynamics which affect the dynamical measurements of emissions (Mrosek et al., 2011).

Therefore a model structure is presented which is not affected by sensor dynamics. The presented model consists of a dynamical mean value air path model which is combined with a stationary combustion model. The air path is modelled by a semi-physical approach with lumped parameters and is shortly described in the following section. The combustion process on the other hand is modelled as a stationary black-box model. Models are given for the emissions $\mathrm{NO}_{\mathrm{x}}$, soot and torque. The model structure is shortly described in the section thereafter. Finally for optimisation of a step in the acceleration pedal, both models are connected to simulate the dynamical emissions from the actuator positions to the outputs $\mathrm{NO}_{\mathrm{x}}$, soot and torque. The regarded actuator positions are the high-

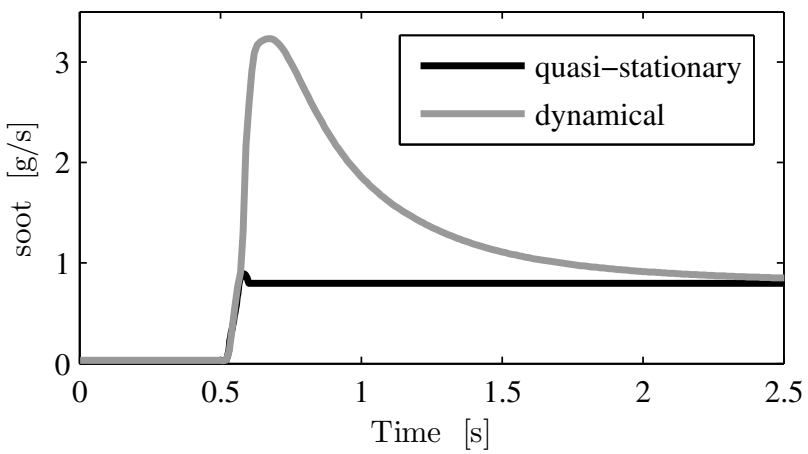

Figure 1. Quasistationary and dynamical simulation of the soot emissions for a ramp in injection mass $\left(u_{\mathrm{inj}}=\right.$ $15-30 \mathrm{~mm}^{3} /$ cyc from $t=0.5-0.6 \mathrm{~s}$ for a basic calibration.

pressure egr valve $s_{\text {egr }}$, the position of the turbochargers guide vanes $s_{\mathrm{t}}$ and the combustion feature crank angle of $50 \%$ mass fraction burnt $\varphi_{\mathrm{Q} 50}$, which mainly depends on the crank angle of the injection events (main and pilot injections).

The optimisation results give a benchmark for future design of engine controllers. The results are therefore compared to a nonlinear control of the air path. As the presented approach optimises the emissions with respect to their steady state values, the results can directly be compared to approaches controlling the emissions as is shown in Alfieri (2009). As the optimisation of a step in acceleration requires a priori knowledge of the acceleration pedal trajectory, the results can only partly be transferred to the ECU. However insights about the formation of emis- 


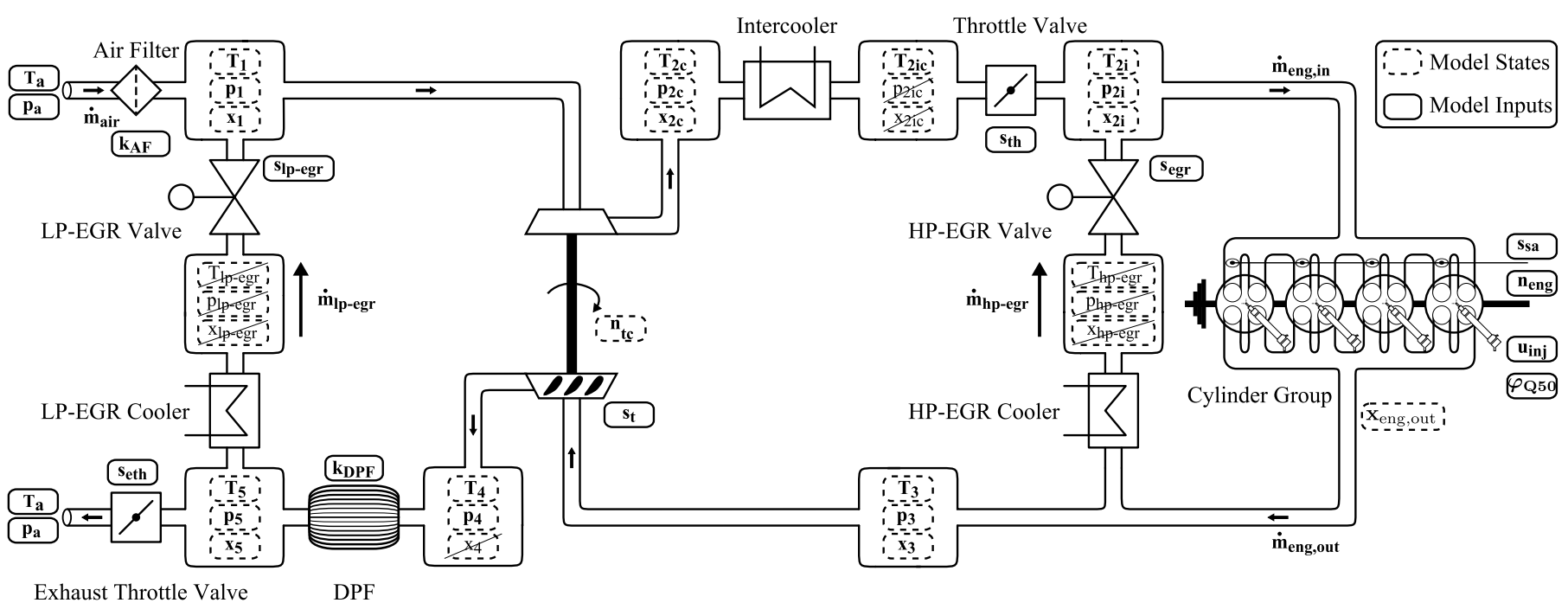

Figure 2. Mean value model of the air path using a lumped parameter approach. Crossed out states in the storages are neglected.

sions at transient driving are given which allow conclusions for the design of feed-forward control strategies.

The optimisation of transient driving is also the objective of future work. Hafner et al. (2000) investigated the optimisation of transient engine feed-forward control. The fuel consumption as well as the emissions $\mathrm{NO}_{\mathrm{x}}$ and soot are modelled by dynamical LOLIMOT models with the actuator signals as model inputs. Enhancements in the feed-forward control of air path actuators are achieved. The approach in Benz et al. (2010) is similar to the one presented here. An air path model is connected to a combustion model which models the emissions based on a grid map for stationary engine operation and correction terms for transient engine operation. Optimisation is done for the actuators $s_{\mathrm{egr}}, s_{\mathrm{t}}$, the start of injection and the common rail pressure. The optimisation criterion minimises the $\mathrm{cu}-$ mulated emissions. An alternative optimisation criterion is applied in Alberer and del Re (2009), where the deviation of the emissions from the calibrated steady state emissions is penalised. The optimisation is done without a model directly on the engine testbed. In the following a similar criterion is used but the optimisation is model-based with a dynamical air path model and a stationary emission model.

\section{DYNAMICAL MEAN VALUE AIR PATH MODEL}

The air path is modelled with a lumped parameter approach. The individual components (intercooler, turbocharger, etc.) are modelled separately and are connected via storages to an overall mean value model. Engine testbed measurements are utilised to parametrise the submodels. Physically complex relations such as the exhaust gas temperature or the volumetric efficiency are modelled as black-box models. The model structure can therefore be regarded as semi-physical. The model states and inputs are shown in the sketched engine air path in Fig. 2. Model inputs are the ambient pressure $p_{\mathrm{a}}$ and temperature $T_{\mathrm{a}}$ and the actuator positions $s$. Model states are the temperatures $T_{i}$, the gas compositions $x_{i}$ and the pressures $p_{i}$ in the storages $i$ as well as the turbocharger speed $n_{\mathrm{tc}}$. The crossed out states are neglected to reduce the model complexity. The values of the gas compositions $x_{i}$ range from 1 for fresh air with $21 \%$ oxygen to 0 for burnt gas containing $0 \%$ oxygen. The gas composition after combustion is given by

$$
\begin{aligned}
x_{\text {eng,out }} & =\frac{m_{\text {air }, \text { eng }}-m_{\text {air, burnt }}}{m_{\text {gas,total }}} \\
& =\frac{x_{2 \mathrm{i}} m_{\text {eng,in }}-u_{\text {inj }} \rho_{\text {Diesel }} L_{\text {st }}}{m_{\text {eng,in }}+u_{\text {inj }} \rho_{\text {Diesel }}}
\end{aligned}
$$

The air path model covers the major dynamics of the Diesel engine. It also contains simple actuator models, whereas the actuator time constants are relatively small compared to the system time constants. They do however have an effect on the optimisation result. The system time constants are mainly introduced by the turbocharger and the egr paths. More detailed descriptions and discussions of the air path model can be found in Mrosek et al. (2009) and Mrosek and Isermann (2010). Assuming the combustion as a batch process the emission formation mainly depends on the cylinder charge at intake valve closing and the injections (Sequenz et al., 2010; Mrosek et al., 2010). Therefore the intake system dynamics determine the major dynamics of emission formation.

For the presented work the low-pressure egr valve $s_{\text {lp-egr }}$ is kept closed, to keep the dimensionality low. Thus the regarded actuators for the optimisation are the position of the high-pressure egr valve $s_{\text {egr }}$, the position of the guide vanes of the turbocharger $s_{\mathrm{t}}$ and the crank angle of $50 \%$ mass fraction burnt $\varphi_{\mathrm{Q} 50}$. The latter one has a direct influence on the combustion and therefore on the formation of emissions. Hence it is also included in the combustion model which is presented in the following section. It shall be mentioned that $\varphi_{\mathrm{Q} 50}$ is adjusted by the timing of the main and the pilot injections. It can therefore either be modeled by these inputs or be calculated with an indicating system using in-cylinder pressure measurements. 


\section{STATIONARY COMBUSTION MODEL}

At first a transformation of the emissions is presented. This is necessary as emissions are usually measured as concentration though the cumulated emission mass is relevant for the legislative regulations. Emissions can be measured in parts per million $c_{\mathrm{x}}$ and can then be transformed to a emission flow rate by

$$
\dot{m}_{\mathrm{x}}[\mathrm{mg} / \mathrm{s}]=c_{\mathrm{x}}[\mathrm{ppm}] \frac{m_{\mathrm{mol}, \mathrm{x}}[\mathrm{g} / \mathrm{mol}]}{m_{\mathrm{mol}, \mathrm{exh}}[\mathrm{g} / \mathrm{mol}]} \dot{m}_{\text {exh,out }}[\mathrm{kg} / \mathrm{s}] .
$$

At this $m_{\mathrm{mol}, \mathrm{x}}$ is the molar mass of the measured emissions and $m_{\text {mol,exh }}$ is the molar mass of the exhaust gas which can be simplified to the molar mass of air $(28.96 \mathrm{~g} / \mathrm{mol})$. $\dot{m}_{\text {exh,out }}$ is the tailpipe gas mass flow rate and does therefore not include the recirculated exhaust gas. For $\mathrm{NO}_{\mathrm{x}}$ the molar mass is given as $\mathrm{NO}_{2}$ equivalent $(46.01 \mathrm{~g} / \mathrm{mol})$, see European Commission (2007). The soot concentration $c_{\text {soot }}$ is measured in $\mathrm{mg} / \mathrm{m}^{3}$ and needs therefore be transformed by

$$
\dot{m}_{\text {soot }}[\mathrm{mg} / \mathrm{s}]=\frac{c_{\text {soot }}\left[\mathrm{mg} / \mathrm{m}^{3}\right]}{\rho_{\text {exh,meas }}\left[\mathrm{kg} / \mathrm{m}^{3}\right]} \dot{m}_{\text {exh,out }}[\mathrm{kg} / \mathrm{s}],
$$

whereas $\rho_{\text {exh,meas }}$ is the density of the gas in the measuring cell. Applying these transformations the emissions can be modelled as mass flow rates which are more meaningful for an optimisation then the concentrations.

For a simplified emission model, the combustion process is regarded as a batch process. The in-cylinder conditions at intake valve closing and the combustion feature $\varphi_{\mathrm{Q} 50}$ determine the outcome of that batch process. Thus a mean value model can be applied which only depends on the states of the air path before the cylinder (air mass per cycle $m_{\text {air }}$, intake pressure $p_{2 \mathrm{i}}$ and intake temperature $\left.T_{2 \mathrm{i}}\right)$, the engine operation point (engine speed $n_{\mathrm{eng}}$ and injection quantity $u_{\text {inj }}$ ) and a combustion feature (crank angle of $50 \%$ mass fraction burnt $\left.\varphi_{\mathrm{Q} 50}\right)$. As the formation of soot mainly depends on the local availability of oxygen, the gas composition after combustion $x_{\text {eng,out }}$ is advantageous for the soot model. It is therefore also included as alternative input to $m_{\text {air }}$.

As the process is regarded as a batch process, a stationary model structure is applied, see Fig. 3. It consists of several local polynomial models (LPM) which are superimposed to a global model by means of weighting functions (a linear interpolation). The weighting functions are determined by the operation point given as z-regressors. The x-regressors are the remaining model inputs. For the parameters of the local polynomial models, selection strategies are applied which enable an adaption to the process nonlinearities and increase the model quality, see Sequenz et al. (2009).

The local models are parametrised with experimental data centred in the same operation point as the model centres. For global model validation measurements are centred in between the local models, see Fig. 4. The figure shows an extract of the taken measurements which cover the relevant engine operation points for commonly used driving cycles. In each local model about 250 measurements are taken to parametrise the local models. Detailed descriptions of the emission models with training and validation results for stationary and dynamical data can be found in Sequenz et al. (2010) and Mrosek et al. (2010).

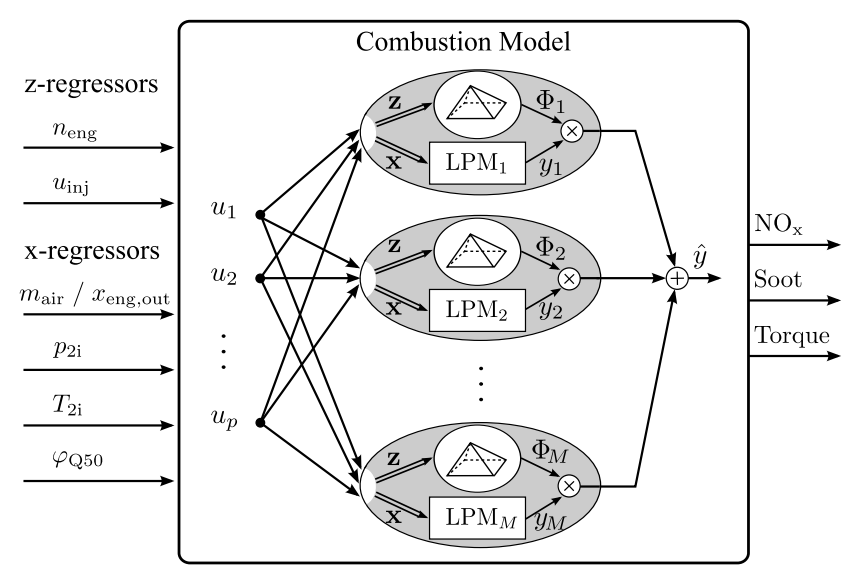

Figure 3. Network structure consisting of local polynomial models (LPM) with x-regressors as model inputs and weighting functions determined by the z-regressors.

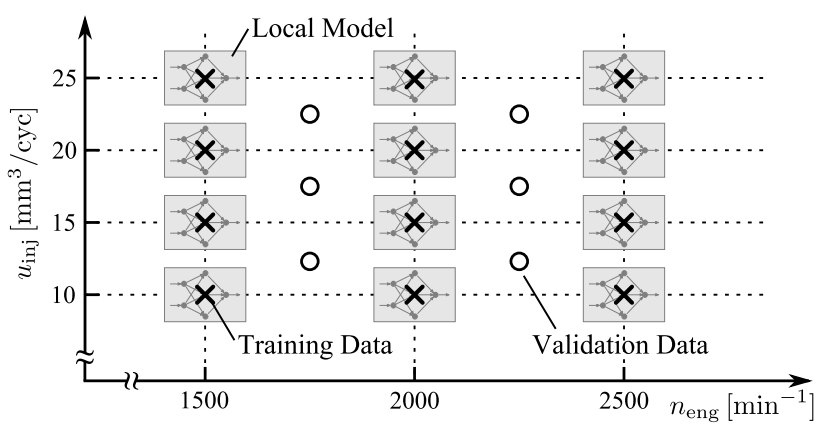

Figure 4. Extract of the distribution of local polynomial models with training and validation data in the engine operation range.

A main advantage of the presented model structure is its independence to measurement dynamics. Mrosek et al. (2011) show that quantities measured by emission sensors are affected by a variable dead time and a variable time constant, mainly depending on the volume flow rate in the exhaust system. These dynamics are not neglectable in relation to the system dynamics and can not be eliminated from the measurements. Therefore the parametrisation of the combustion model is done with stationary data. Thus all measurement dynamics are eliminated. For simulation purpose the system dynamics are covered by the dynamic air path model. A connection of these models enables a simulation of the dynamic behaviour from the actuators to the regarded model outputs.

The connection between the dynamic air path model and the stationary emission model is shown in Fig. 5. For optimisation the actuator positions of the high-pressure egr valve $s_{\text {egr }}$ and the vgt-actuator $s_{\mathrm{t}}$ are considered. Further the crank angle of $50 \%$ mass fraction burnt $\varphi_{\mathrm{Q} 50}$ is considered. Intermediate variables are the air path states $p_{2 \mathrm{i}}, T_{2 \mathrm{i}}$ and $m_{\text {air }}$ respectively $x_{\text {eng,out. In combination }}$ with $\varphi_{\mathrm{Q} 50}$ these account as model inputs for the mean value combustion model. Finally $\mathrm{NO}_{\mathrm{x}}$, soot and torque are outputs of the combustion model. The highlighted printed variables in Fig. 5 indicate the variables regarded for optimisation. 


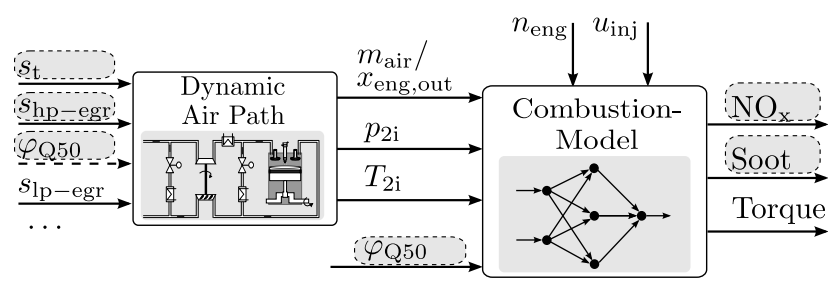

Figure 5. Block diagram of the combined air path and combustion model. The model simulates the emissions and the torque with respect to the engine actuators. Variables regarded for optimisation are highlighted.

\section{MODEL-BASED OPTIMISATION OF THE EMISSIONS FOR A STEP IN ACCELERATION}

In the following section the optimisation criterion is defined. Then the trajectories for the actuators are optimised based on this criterion.

\subsection{Optimisation criterion}

The objective function is the basis for all optimisations. Stationary optimised operation points are usually compromises of several objectives (emissions, fuel consumption, noise, etc.). Therefore a weighted minimisation regarding only the cumulated emissions does not yield to the desired solution. On the other hand a formulation of a criterion considering all relevant outputs would increase the complexity of the optimisation problem. Therefore an objective function is chosen with respect to the stationary optimised emission values

$$
\begin{aligned}
V= & \int_{0}^{T_{\text {end }}}\left(\frac{\left|\mathrm{NO}_{\mathrm{x}}(t)-\mathrm{NO}_{\mathrm{x}, \text { stat }}\right|}{\mathrm{NO}_{\mathrm{x}, \text { stat }}}\right)^{2} \\
& +\left(\frac{\left|\operatorname{soot}(t)-\operatorname{soot}_{\text {stat }}\right|}{\text { soot }_{\text {stat }}}\right)^{2} d t .
\end{aligned}
$$

Thus the deviations of the simulated emissions $\mathrm{NO}_{\mathrm{x}}(t)$ and $\operatorname{soot}(t)$ to the stationary calibrated emissions $\mathrm{NO}_{\mathrm{x}, \text { stat }}$ and soot $_{\text {stat }}$ are penalised. Hence a fast settling time to the stationary calibrated states is enforced. There are especially no further parameters necessary to weight the emissions as both have to follow their desired trajectory.

The optimisation of the trajectories requires an optimisation of every regarded actuator for all time steps. Thus for a discretisation in $N$ time steps and the 3 actuators $s_{\mathrm{egr}}, s_{\mathrm{t}}$ and $\varphi_{\mathrm{Q} 50}$ to be optimised, the problem is of $3 N$ dimensionality. Hence already a rough discretisation can result in numerical difficulties. Therefore a discretisation in $N=10$ steps is chosen for an optimisation period of $3 \mathrm{~s}$. The discretisation can be regarded as trade-off between variety of actuator trajectories and computing time.

Previous approaches of transient optimisation assumed constant values for the actuators in between the discretised time steps. Thus the optimal trajectory results in a step function (e.g. Benz et al. (2010)). In the following an interpolation between the optimised actuator values is applied. Due to the increase of flexibility compared to a step function this appears to be advantageous. For interpolation piecewise cubic hermitian polynomials as described in Moler (2004) are applied. They are more flexible than simple linear interpolations but do less tend to oscillations than general spline interpolations. Furthermore the resulting trajectories are smooth and thus the actuators are not actuated by steps in the setpoint.

Besides the discretisation in $N=10$ steps, finer rasters have been applied. The initial values for optimisation have been taken from the optimisation results for $N=10$. As the results for the finer rasters showed no significant improvements, the results for $N=10$ are presented in the following.

\subsection{Optimisation of a Step in Acceleration}

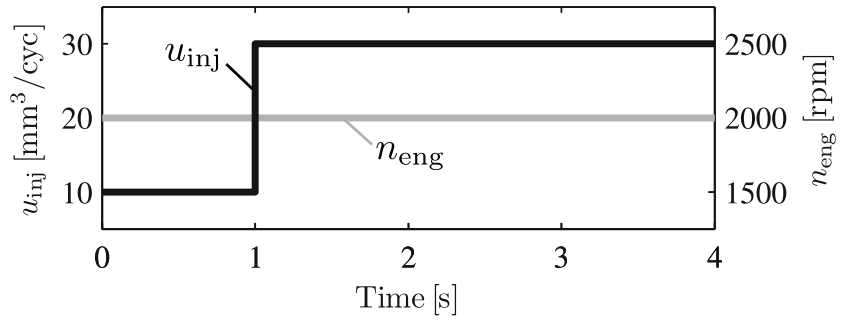

Figure 6. Step in the desired injection quantity from $u_{\text {inj }}=10$ to $30 \mathrm{~mm}^{3} /$ cyc with constant engine speed $n_{\mathrm{eng}}=2000 \mathrm{~min}^{-1}$.

For optimisation of the transient behaviour, a step in acceleration is considered. Therefore the engine speed is kept constant at $n_{\mathrm{eng}}=2000 \mathrm{~min}^{-1}$ and the injection quantity increases at $t=1 \mathrm{~s}$ from $u_{\text {inj }}=10$ to $30 \mathrm{~mm}^{3} /$ cyc, see Fig. 6. For this step the utilised local combustion model changes instantaneously from the local model at $n_{\mathrm{eng}}=2000 \mathrm{~min}^{-1}, u_{\mathrm{inj}}=10 \mathrm{~mm}^{3} /$ cyc to the local model at $n_{\mathrm{eng}}=2000 \mathrm{~min}^{-1}, u_{\mathrm{inj}}=30 \mathrm{~mm}^{3} /$ cyc. The local model inputs $\left(p_{2 \mathrm{i}}, T_{2 \mathrm{i}}\right.$ and $m_{\text {air }}$ respectively $\left.x_{\text {eng,out }}\right)$ are determined by the dynamic air path model and are therefore affected by the system dynamics. These dynamics do again depend on the air path actuators affecting the states of the gas entering the cylinder.

At first the actuators are feed-forward controlled, as can be seen in Fig. 7. The actuators in the upper two plots are changed instantaneously from their stationary calibrated value at $\left(n_{\text {eng }}=2000 \mathrm{~min}^{-1}, \quad u_{\text {inj }}=10 \mathrm{~mm}^{3} /\right.$ cyc $)$ to their stationary value at $\left(n_{\text {eng }}=2000 \mathrm{~min}^{-1}, u_{\text {inj }}=\right.$ $30 \mathrm{~mm}^{3} /$ cyc). The dynamics of the actuators can be seen in the actuator positions (topmost plot). The air path states follow with significant dynamics, see the middle two plots. This is especially apparent for the intake pressure $p_{2 \mathrm{i}}$ which is even after $3 \mathrm{~s}$ not settled. The air mass per cycle $m_{\text {air }}$ follows with the same dynamics apart from an instant peak at $t=1 \mathrm{~s}$. The effects of these dynamics can also be seen in the gas composition after combustion $x_{\text {eng,out }}$. Significant low values are observable after the step in acceleration which implies a lack of air for the combustion. The effect from this can well be seen in the soot emissions (bottom plot). As the emission model is operated in extrapolation region the output is limited to the maximum value observed at model training. After $1.5 \mathrm{~s}$ the soot emissions decay to the stationary calibrated value. The $\mathrm{NO}_{\mathrm{x}}$ emissions approach their stationary value from the other side which is expected by the $\mathrm{NO}_{\mathrm{x}}$-soot trade-off. 

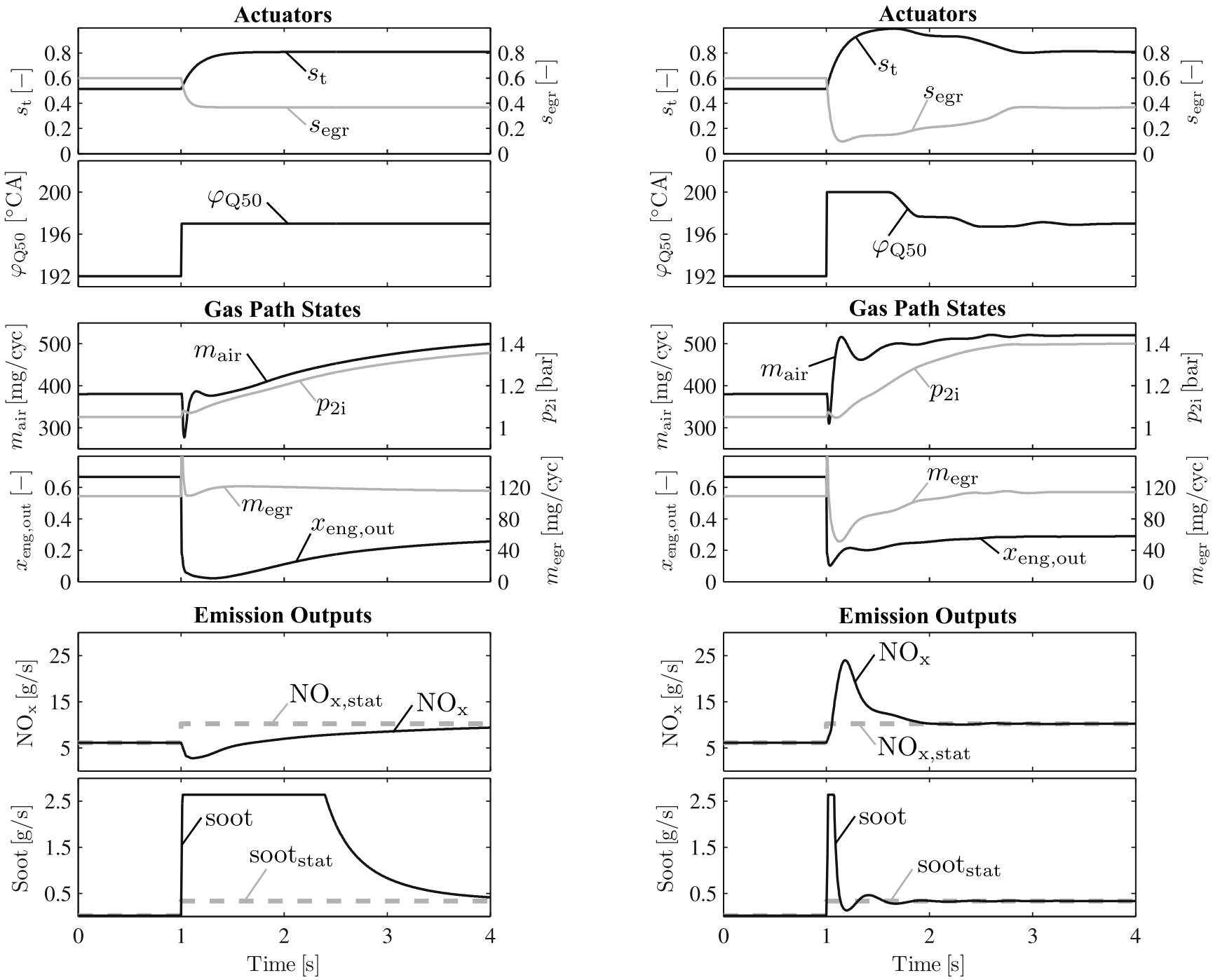

Figure 7. Actuator positions, air path states and emissions for a non optimised step in injection quantity from $u_{\text {inj }}=15$ to $25 \mathrm{~mm}^{3} /$ cyc.

In Fig. 8 the optimised trajectory for the same step in injection quantity is shown. It is optimised by an iterative optimisation algorithm with the objective function as described in section 4.1. The actuator positions are optimised subsequently starting with the positions at $t=1 \mathrm{~s}$. After one iteration the optimisation is started from the beginning with the initial values taken from the previous iteration. From the topmost plot it can be seen that it is advantageous to overshoot the calibrated stationary value for the turbocharger guide vanes $s_{\mathrm{t}}$ to reduce the settling time of the intake pressure. The guide vanes are closed for the first few milliseconds. Due to actuator dynamics the fully closing requires about $0.5 \mathrm{~s}$. The hp-egr valve is not fully closed. Again the closing is affected by actuator dynamics. After 0.1s the air mass flow increases such that the egr-valve is kept partly open to prevent a larger overshoot of the $\mathrm{NO}_{\mathrm{x}}$ emissions. Further the crank angle of $50 \%$ mass fraction burnt is trimmed to a later combustion to further lower the soot and $\mathrm{NO}_{\mathrm{x}}$ emissions. It can be seen in the middle two plots of Fig. 8 that the air path states settle considerably faster than in the non optimised case. Especially $x_{\text {eng,out }}$ has significant higher values. The lack

Figure 8. Actuator positions, air path states and emissions for an optimised step in injection quantity from $u_{\mathrm{inj}}=$ 15 to $25 \mathrm{~mm}^{3} / \mathrm{cyc}$

of air is significantly shorter which can also be observed by the peak in soot emissions. The long soot peak could thus be reduced to a narrow peak of soot. However the fast delivery of fresh air has a negative effect on the $\mathrm{NO}_{\mathrm{x}}$ emissions as can be seen by the overshoot of the $\mathrm{NO}_{\mathrm{x}}$ emissions.

To further avoid the soot peak the injection mass has to be limited. This can either be done by shaping the setpoint of the injection mass with a first order lag or by a smoke limitation, as presented in Sequenz et al. (2011). The desired injection quantity should then still be passed to the controllers such that the actuators can be adjusted accordingly.

The non optimised case assumes that values for the actuators are calibrated for stationary engine operation. Modern engine control units do however incorporate desired values for the air path states and employ a closed loop control to reach these desired values. Therefore in Table 1 the optimised results are compared to a nonlinear closed loop air path control as described in Pfeil et al. (2007). Controlled variables are the air mass flow rate and the intake pressure. 
In the comparison the nonlinear air path control defines the $100 \%$ for the regarded outputs.

Table 1. Comparison of the nonlinear air path control to the spline based optimisation, the step function based optimisation and the non optimised results.

\begin{tabular}{lccc}
\hline & $\mathrm{NO}_{\mathrm{x}}$ & soot & torque \\
\hline spline based & $75.9 \%$ & $84.3 \%$ & $102.1 \%$ \\
step function & $78.8 \%$ & $82.7 \%$ & $101.5 \%$ \\
non optimised & $44.8 \%$ & $403.1 \%$ & $95.8 \%$ \\
air path control & $100.0 \%$ & $100.0 \%$ & $100.0 \%$ \\
\hline
\end{tabular}

The presented optimised results (spline based) show a significant reduction of the $\mathrm{NO}_{\mathrm{x}}$ emissions. This is due to a partly opening of the egr valve during the transient. The soot emissions could also be reduced which is due to a faster acceleration of the turbocharger. Despite the late injection the generated torque is slightly above the torque from a simulation with closed loop air path control. This is mainly due to the faster settling of the air path. It can further be seen that the results for the step function are similar to the spline based trajectory. This is since the atuator dynamics smooth the steps in the setpoints. The time to reach steady state is however significantly longer for the step function approach. The non optimised case is also given for comparison. Especially the long soot peak has a poor effect on the cumulated emissions and do not pay off the lower $\mathrm{NO}_{\mathrm{x}}$ emissions.

\section{CONCLUSIONS}

A model structure to simulate the emissions and the torque from the actuators of the air path to the outputs $\mathrm{NO}_{\mathrm{x}}$, soot and torque is presented. The derived model structure is independent of sensor dynamics as the combustion is modelled separately to the dynamic air path. The dynamics of emission formation are covered by the dynamical air path model which is connected to the stationary combustion model. This combined model structure is applied for the optimisation of a step in acceleration. Based on calibrated stationary emissions, an objective function is defined which forces the emissions to their stationary values. An open loop simulation shows that the settling time of the air path dynamics is significant and poorly affects the soot emissions. The optimisation of the spline interpolated trajectories of the actuators show a smooth characteristic for the actuators and the outputs. For the optimised actuator trajectories the soot peak could significantly be reduced. Furthermore the generated torque could be increased slightly. This pays off the moderate increase of $\mathrm{NO}_{\mathrm{x}}$ emissions.

The presented optimisation results conclude that it is advantageous to overshoot the actuator values to reach steady state faster. This is also achieved by the presented nonlinear air path control. The optimisation further shows that it is superior to not fully close the egr valve during the transients. The slight opening enables a control of the $\mathrm{NO}_{\mathrm{x}}$ emissions. The remaining soot peak can only be avoided by limiting the injection quantity at transients by shaping the injection setpoint or by a smoke limitation.
Due to the significant calculation time the optimisation can not directly be transferred to an ECU. It gives however an insight about the transient formation of emissions and a benchmark for future controller designs.

\section{REFERENCES}

Alberer, D. and del Re, L. (2009). Optimization of the transient Diesel engine operation. In 9th International Conference on Engines and Vehicles.

Alfieri, E. (2009). Emissions-Controlled Diesel Engine. Dissertation, Swiss Federal Institute of Technology Zürich.

Benz, M., Onder, C., and Guzzella, L. (2010). Engine Emission Modeling Using a Mixed Physics and Regression Approach. Journal of Engineering for Gas Turbines and Power, 132, 042803.

European Commission (2007). Regulation (EC) no $715 / 2007$ of the european parliament and of the council. Official Journal of the European Union L171, 50, 1-15. Hafner, M., Schüler, M., Nelles, O., and Isermann, R. (2000). Fast neural networks for Diesel engine control design. Control Engineering Practice, 8(11), 1211-1221.

Moler, C. (2004). Numerical computing with MATLAB. Society for Industrial Mathematics.

Mrosek, M. and Isermann, R. (2010). Dynamic reference value generation for the control of a Diesel engine with hp and lp-egr. In ASME Dynamic Systems and Control Conference.

Mrosek, M., Sequenz, H., and Isermann, R. (2010). Control oriented $\mathrm{NO}_{\mathrm{x}}$ and soot models for Diesel engines. In IFAC Symposium Advances in Automotive Control.

Mrosek, M., Zahn, S., and Isermann, R. (2009). Parameter estimation for physical based air path models of turbocharged engines - an experience based guidance. In 9th International Conference on Engines and Vehicles. SAE, Naples, Italy.

Mrosek, M., Sequenz, H., and Isermann, R. (2011). Identification of emission measurement dynamics for Diesel engines. In 18th IFAC World Congress.

Pfeil, K., Zimmerschied, R., and Isermann, R. (2007). Nichtlineare Identifikation des Luftpfads von aufgeladenen Dieselmotoren und automatisierter AGR-/VTGReglerentwurf. at - Automatisierungstechnik, 55, 352359 .

Sequenz, H., Mrosek, M., and Isermann, R. (2010). Stationary global-local emission-models of a CR-Diesel engine with adaptive regressor-selection for measurements of airpath and combustion. In IFAC Symposium Advances in Automotive Control.

Sequenz, H., Mrosek, M., and Isermann, R. (2011). Model based smoke limitation for the control of turbocharged CR-Diesel engines. In 11th Stuttgart International Symposium.

Sequenz, H., Schreiber, A., and Isermann, R. (2009). Identification of nonlinear static processes with local polynomial regression and subset selection. In Proceedings of the 15th IFAC Symposium on System Identification. 